\title{
A Search for Planetary Transits of the Star HD 187123 by Spot Filter CCD Differential Photometry
}

\author{
T. Castellano ${ }^{1}$ \\ NASA Ames Research Center, MS 245-6, Moffett Field, CA 94035 \\ tcastellano@mail.arc.nasa.gov
}

Received —_ accepted —

Submitted to Publications of the Astronomical Society of the Pacific

\footnotetext{
${ }^{1}$ Also at Department of Astronomy and Astrophysics, University of California, Santa Cr11\%. CA 95064
} 


\begin{abstract}
A novel method for performing high precision, time series CCD differential photometry of bright stars using a spot filter, is demonstrated. Results for several nights of observing of the 51 Pegasi b-type planet bearing star HD 187123 are presented. Photometric precision of $0.0015-0.0023$ magnitudes is achieved. No transits are observed at the epochs predicted from the radial velocity observations. If the planet orbiting $\mathrm{HD} 187123$ at $0.0415 \mathrm{AU}$ is an inflated Jupiter similar in radius to HD 209458b it would have been detected at the $>6 \sigma$ level if the orbital inclination is near 90 degrees and at the $>3 \sigma$ level if the orbital inclination is as small as 82.7 degrees.
\end{abstract}

Subject headings: stars: planetary systems techniques:photometric

\title{
1. Introduction
}

More than two dozen extrasolar planets have been discovered around nearby stars by measuring their Keplerian radial velocity Doppler shifts (see, for example, Marcy et al. (2000)). Two radial velocity teams recently discovered a planet orbiting the star HD 209458 (Henry et al. 1999; Mazeh et al. 2000). The planet has $M_{p} \sin i=0.62 \mathrm{M}_{\mathrm{J}}$ $\left(1 \mathrm{I}_{\mathrm{J}}=1\right.$ Jupiter mass $\left.=2 \times 10^{30} \mathrm{grams}\right)$ and $a=0.046 \mathrm{AU}$, with an assumed eccentricity of zero but consistent with 0.04 (Henry et al. 1999). Photometric observations detected a dimming of this star's light consistent with a planetary transit (Charbonneau et al. 1999; Henry et al. 1999; hereafter, C99 and H99, respectively). This first detection of a transit signal of a 51 Pegasi b-type planet, allows determination of the planet's mass (by knowing the inclination) and radius (from the depth of the transit), giving in this instance. a mass and radius of $0.63 \mathrm{M}_{\mathrm{J}}$ and $1.27 \mathrm{R}_{\mathrm{J}}\left(1 \mathrm{R}_{\mathrm{J}}=1\right.$ Jupiter radius $=7.14 \times 10^{9} \mathrm{~cm}$ and 
$1 \mathrm{M}_{\mathrm{J}}=1$ Jupiter mass $=1.90 \times 10^{30}$ grams) $(\mathrm{C} 99)$ and $0.62 \mathrm{M}_{\mathrm{J}}$ and $1.42 \mathrm{R}_{\mathrm{J}}(\mathrm{H} 99)$. This inflated physical size of the planet is consistent with models for hot extrasolar planets (hereafter called "inflated Jupiters"(Guillot et al. 1996)). 10\% of short period planets (hereafter called 51 Pegasi b-type planets) are expected to transit solar-sized stars.

Doppler measurements from the Keck HIRES spectrometer of the star HD 187123 indicate the presence of a short period planet (Butler et al. 1998). Transit photometry can yield the mass and radius of this planet if the orbit is viewed nearly edge on. The radial velocity data allows the transit time to be predicted to within the accuracy of the determination of the planet's orbital period. The planet orbiting HD 187123 has $M_{p} \sin i=0.52 \mathrm{M}_{\mathrm{J}}$ and should be inflated like the planet orbiting HD 209458 since they have comparable semimajor axes $(0.0415 \mathrm{AU}$ and $0.0457 \mathrm{AU})$. A maximum central transit depth of 0.018 magnitudes would be produced for an adopted stellar radius of $1.14 \mathrm{R}_{\odot}$ $\left(1 \mathrm{R}_{\odot}=1\right.$ Solar radius $=6.96 \times 10^{10} \mathrm{~cm}$ and $1 \mathrm{M}_{\odot}=1$ Solar mass $=1.99 \times 10^{33}$ grams $)$. Photometric precision of better than 0.006 magnitudes is required for a statistically significant detection (at the $3 \sigma$ level for the most favorable transit geometry). The goal for this work was photometric precision of 0.001 magnitude measured over at least the duration of a central transit of a 51 Pegasi b-type planet of a solar like star (approximately 3 hours). In this work, phtometric precision is defined as the actual measured standard deviation of the differential magnitude measured over the duration of the observations.

\section{Differential Photometric Method}

Differential stellar photometry, the measurement of the difference in brightness of two or more stars has several advantages for the detection of planetary transits. Differential photometry is a simple technique that does not require reduction to a standard magnitude system by the use of separately observed standard stars. It is the most accurate technique 
for measuring small changes in brightness (Henden \& Kaitchuk 1990), and if the stars to be compared are near one another on the celestial sphere and similar in color, the first order (airmass dependent only) extinction and the second order (color dependent) extinction corrections are unnecessary. Traditionally, differential photometry is performed with a single channel photometer, a non-imaging instrument that measures a single star's brightness.

A differential measurement then consists of a pair of measurements of two stars taken sequentially through two different values of airmass. In contrast, Charge Coupled Device (CCD) differential photometry allows the simultaneous measurement of the brightness of all stars in a given field through virtually identical values of airmass. Differential CCD photometry has been demonstrated to achieve precision of 0.0008 magnitudes for a 9 hour time series with 1-meter class telescopes (Gilliland 1992). Differential photometry is robust to small changes in atmospheric transparency (i.e., light clouds) and the efficiency of the instrumentation (such as that caused by optical vignetting). The differential magnitude is then defined as $\delta v=-2.5 \times \log \frac{\text { ObjectStarFlux }}{\text { ComparisonStarFlux }}$ where $\delta \mathrm{v}$ is the differential magnitude in the $\mathrm{V}$ band. The first order extinction is given by $\mathrm{v}_{0}=\mathrm{v}-\mathrm{a}_{\mathrm{v}} \times X$ where $\mathrm{v}_{0}$ is the magnitude of the object star that would be measured in the absence of the Earth's absorbing atmosphere, $X$ is the airmass, $\mathrm{a}_{\mathrm{v}}$ is the $V$ band first order extinction coefficient in magnitudes per unit airmass and $v$ is the measured magnitude in the $V$ band. The first order extinction coefficient $a_{v}$ is usually determined nightly by observing a star through a range of airmasses. Second order extinction depends on the color difference beween the object star and a comparison star and is defined by the equation $v_{0}=\mathrm{v}$ $\mathrm{a}_{\mathrm{v}} \times X-\mathrm{b}_{v} \times(B-V) \times X$ where $\mathrm{b}_{\mathrm{v}}$ is the second order extinction coefficient in units of magnitudes per airmass per unit difference in $B-V$ color.

The Keck radial velocity survey of Marcy et al. has chosen relatively bright stars (few stars in the survey are as dim as 10 magnitudes) in order to obtain the high signal to noise spectra necessary to determine radial velocities with $3 \mathrm{~m} / \mathrm{s}$ precision in short integration 
times. There are only several hundred thousand stars as bright as 10th magnitude on the 41,253 square degrees that make up the celestial sphere, or approximately 5 bright stars per square degree. The comparably bright comparison stars required for standard stellar differential photometry are therefore lacking in any reasonably-sized CCD field of view. It was decided to overcome this comparison star scarcity problem by emploving a neutral density spot filter to reduce the flux from the object star relative to the comparison stars, rather than attempting precision differential measurements over a large field (Borucki et al. 2000; Brown et al. 1999). By using a spot filter, placed near the focal plane and positioned to attenuate the light from the bright object star, integration times on the order of several minutes are possible for the stars that have known planetary companions. For the results reported herein, 250-second integrations are required to suppress atmospheric scintillation noise to the 0.0007 magnitude level (Young 1974). For the special case of HD 187123, its proximity to the galactic plane in the constellation of Cygnus and its relative faintness $\mathrm{m}_{\mathrm{v}}=7.9$ makes the likelihood of suitable nearby comparison stars much higher. A backside-illuminated and thinned CCD was selected because these high quantum efficiency devices are less sensitive to intra-pixel sensitivity variations (Buffington et al. 1991) than frontside-illuminated devices. For frontside-illuminated CCDs, photons must pass through the polysilicon parallel transfer electrodes running in the row direction. These electrodes are used to clock charge out to the serial output registers. In a frontside-illuminated device the photon have to pass through these structures which are opaque to some wavelengths of visible light and lead to spatial quantum efficiency variations within each pixel. Backside-illuminated devices accept photons directly into the bulk silicon from the "backside" of the CCD where no parallel transfer electrodes are present.

The filter used for this work was fabricated by the Lick Observatory optical shop from a $5 \mathrm{~mm}$ core cut from a Schott ncutral density filter of NG 3 glass and nominal thickness $2.00 \mathrm{~mm}$ (giving $8.1 \times 10^{-3}$ transmittance, or 5.23 magnitudes of attenuation) and placed 
in a $5 \mathrm{~mm}$ hole bored in a second Schott neutral density filter of $2.00 \mathrm{~mm}$ thick NG12 glass (giving 0.79 transmittance, or 0.26 magnitudes of attenuation). By selecting this construction method, differences in the thickness and index of refraction of the two filter components that might lead to focus differences between the object and comparison stars were minimized. Room temperature vulcanizing silicon rubber was used as the adhesive to join the two components.

\section{Observations}

The Anna L. Nickel 40" telescope of the University of California's Lick Observatory, Mount Hamilton, California was used to make measurements of HD 187123 on the nights of September 5 and September 8, 1999. The detector employed was a $1024 \times 1024$ pixel SITE backside-illuminated and thinned CCD mounted at the cassegrain focus. The read noise was 1.7 electrons per pixel. The plate scale was 0.3 "per pixel. Table 1 lists the Journal of Observations. The ephemeris of transit centers for HD 187123 (assuming a circular orbit, Geoff Marcy, personal communcication) is $T_{c}=2451374.046+n \times(3.096489)$ where $T_{c}$ is the time of transit center in Julian days and $n$ is an integer. The uncertainties in the transit center reference epoch and period are 0.01 day and 0.003 days respectively:

\section{Data Reductions}

CCD frame bias was removed by the Lick data system at the time of exposure. Images were stored to hard disk during observing and periodically transferred via file transfer protocol (FTP) to a laptop computer. Commercial compact disc writing software was then used to archive each night's data to a separate CD-ROM before the end of an individual run. The laptop was also used to view images and perform simple data culality checks. 
NOAO IRAF was used for image trimming (to remove the overscan region) and CCD calibration (flat fielding). Flat fields were constructed from median combining 17 "dome" exposures using incandescent lamps (Stone 1992) illuminating the inside of the telescope dome. Twilight flats were taken but not used due to the difficulty of obtaining sufficient counts under the spot during the relatively brief summer twilight. The spot filter design resulted in an achieved factor of 25 attenuation for objects under the spot. Ths resulted in a factor of 25 fewer counts per pixel under the spot than in the background in all the flat field frames. Since the typical maximum counts in the background was 20,000 (chosen to be safely below the analog to digital converter saturation level and any non-linear response regime of the $\mathrm{CCD}$ ), 800 - 1000 digital counts per pixel were typically obtained under the spot. To achieve photometric precision of 0.001 magnitude requires the Poisson noise $\sigma$ to be less than 1 part in one thousand $(\sigma=\sqrt{N})$, where $N$ is the number of photoelectrons collected per pixel in the combined flat field exposures, implying one million photoelectrons are required per pixel. In practice a less stringent requirement than this is realized since each star's brightness is measured over apertures no smaller than 6 pixels in radius or an area of about 113 pixels. For a gain of 4.4 electrons per analog to digital unit (ADU) the average integrated flux over the aperture in a typical flat field pixel under the spot was $6.8 \times 10^{6}$ ADUs. This results in a contribution to the errors of 0.0004 magnitudes from the Poisson noise in the flat fields. For stars not under the spot the contribution to the errors from the Poisson noise due to the flat fielding process is small compared to the errors introduced from Poisson noise in the flux from the stars themselves. No cosmic ray removal or bad pixel masking was performed. The IRAF routines IMEXAM and XIMTOOL were used to select object and comparison stars interactively and to determine the necessary aperture sizes. All stars were given the same aperture size for the entire night and an initial stellar coordinates file was constructed as input to the aperture photometry routine PHOT in the APPHOT package. Recentering of the apertures was performed for each frame. The 
PHOT routine "mode" option was selected for background determination since this method is less sensitive to hot pixels. IRAF TXDUMP was used to produce tables of position, fluxes, errors, and the universal times of each exposure, for each star in each frame. A computer program written in Interactive Data Language (IDL) was used to read the IRAF output files, reduce the data to differential magnitudes for each $s$ tar in each frame, and plot the results versus universal time. Diagnostics of flux, centroid position, and background flux for each star were also plotted and examined.

For CCD differential photometry, differential (or first order) extinction is due to differences in airmass between the object and comparison stars in a single exposure. Differential extinction is smallest at low values of airmass (at the zenith, for example) and is largest at high values of airmass (at the horizon, for example). The typical extinction coefficients $a_{v}$ found at Lick were 0.36 to 0.47 magnitudes per airmass in the $V$ band. The maximum angular distance between the object star and any single comparison star was 3.54 arc minutes. Combining these extinction coefficients and angular distances with a maximum airmass of 1.5 , results in a maximum predicted differential extinction of 0.0006 magnitudes. In practice, comparison stars are arranged around the object star so that some will have a greater airmass than the object star and some less. Generally, for low values of airmass, differential extinction is much less. In fact, differential extinction contributes less than a micromagnitude to the errors in the differential magnitudes at the meridian for stars at the declination of HD 187123. For these reasons, first order extinction was ignored.

Because of the long exposure times possible with the spot filter (250 seconds), dozens of suitable comparison stars down to about a magnitude of 12 were available within the 5 arc minute square field of view. Exposures were taken in the $\mathrm{B}$ and $\mathrm{V}$ filters and 4 stars with a $B-V$ color within 0.1 of the object star (HD 187123) were chosen as comparison stars. Choosing similar color comparison stars minimizes the second order extinction effect which 
varies with the airmass $X$ and the difference in $B-V$ color. The size of the second order extinction correction is typically 0.03 magnitude for a $B-V$ difference of 1 over a unit change of airmass (Janes 1996). For the observations reported herein, the largest change in airmass over the night was 0.40 and the largest $B-V$ difference was -0.067 (Star 3) which translates to a color-dependent extinction of -0.0008 magnitudes using the Janes estimate.

The differential magnitude of each star relative to the other 4 was calculated for each frame for the 2 nights of observation of HD 187123. For example, star 2 was compared to the sum of the flux from stars 1,3,4 and 5. The standard deviation of this differential measure yields an estimate of the variability of the comparison stars (Table 2). There is no cvidence for variability of comparison stars 2,4 , and 5 within the errors of the measurements. Star 3 does show a difference in the mean differential magnitude between the two nights at the 2.2 $\sigma$ level. The small change in mean differential magnitude from night to night for each star is also an indication of the photometric stability of the system. The 2 data sets were taken with one spot installation and reduced using the same normalized master flat fields.

Even though the first order extinction (due to airmass differences beween the object and comparison stars) and second order extinction (due to color differences beween the object and comparison stars) corrections have been shown to be small compared to the 0.001 magnitude precision goal, there was evidence for a residual linear trend in the data. A linear fit was calculated and subtracted from the differential magnitude of HD 187123. This residual linear component has a slope of 0.0005 and 0.0004 magnitudes per hour for the nights of September 5, 1999 (U. T.) and September 8, 1999 (U. T.) respectively. Figures 1 and 2 show the data after the linear trend is removed. Subtraction of this linear trend improved the measured precision (Table 1). 


\section{Discussion}

Keplerian Doppler variations with a period of 3.1 days of the solar like star HD 187123 were first reported by Butler et al. (1998) The Doppler variations imply an orbiting companion of mass $0.52 \mathrm{M}_{J}$ or larger in a near circular $0.0415 \mathrm{AU}$ orbit. The radial velocity method alone cannot determine the inclination of the orbit, hence the ambiguity in the mass. HD 187123 is a solar twin with a visible spectrum that differs from the sun's by less than 1\%, (Butler et al. 1998). but is slightly more luminous than the sun at $\mathrm{L}=1.35$ $\mathrm{L}_{\odot}$ and has a slightly higher effective temperature of $5830 \mathrm{~K}$ (Gonzalez et al. 1996). This combination of effective temperature and luminosity yields a radius of $1.14 \mathrm{R}_{\odot}$ (Table 3 ). At an orbital distance of just $0.0415 \mathrm{AU}$ the equilibrium global average effective temperature of the planet around HD 187123 is near $1250 \mathrm{~K}$. It may be that the planet is inflated by the extreme stellar insolation to $1.4 \mathrm{R}_{\mathrm{J}}($ Guillot et al. 1996). If this is the case, then the transit depth is increased by a factor of about two over that produced by a Jupiter radius planet. Central transits have maximum depths given by the simple ratio of the planets to the stars disk areas. Non-central transits have smaller depths at all wavelengths due to limb darkening. Since HD 187123 is a solar twin (Butler et al. 1998), it is expected to follow a solar limb darkening law (Allen 1976). At an inclination of 82.7 degrees, solar like limb darkening reduces the transit signal to 0.55 that of a central transit in the $\mathrm{V}$ band. At this value of inclination, the transit duration is 0.31 that of a central transit. This value of inclination was chosen as a limiting case because at this inclination angle, $95 \%$ of orbital inclinations that will produce a transit are included. Figures 1 and 2 demonstrate that transits of inflated Jupiters would have been easily detected if the orbital inclination is beween 82.7 and 90 degrees and that Jupiter-sized planets would be detected only for inclinations very near 90 degrees (Table 4). The simulated idealized transits in Figures 1 and 2 include the effect of solar like limb darkening in the transit depth determination but do not include the effect of limb darkening on the transit ingress and egress profiles. A 
giant terrestrial planet with a mass of $0.5 \mathrm{M}_{\mathbf{J}}$ has a radius of $0.31 \mathrm{R}_{\mathbf{J}}$ (Guillot et al. 1996) and would not have been photometrically detectable in transit with the precision reported herein.

\section{Conclusion}

The recent detection of a planetary transit of the star HD 209458 by Charbonneau et al. and Henry et al. amply demonstrates the utility of transit photometry in determining the mass and radius of extrasolar planets. Accurate determination of the radius and atmospheric structure of this and other 51 Pegasi b-type planets as required to constrain structural models of extrasolar giant planets will depend on continued precision photometry with high time resolution. A novel method for performing this high precision, time series CCD differential photometry of bright stars, has been demonstrated. No transits are observed at the epochs predicted from the radial velocity observations for the star HD 187123.

The author wishes to thank S. Vogt and G. Marcy, for their generous encouragement and advice and L. Doyle, M. Chuang, and H. Deeg for their help with the observing. I also thank A. Misch, R. Stone, and E. Gates for tutorials on the operation of the Nickel telescope and D. Hilyard and B. Brown for the fabrication of the spot filter. Lick Observatory staff members E. Stateler and D. Severinsen were frequently called upon to install or remove the spot filter in response to last minute transit time predictions. These measurements could not have been completed without their rapid response. This work has been supported in part by the NASA Ames Research Center Director's Discretionary Fund and the LC Santa Cruz Davidson Fund. 


\section{REFERENCES}

Allen, C. W. 1976, Astrophysical Quantities, (London: Athlone Press)

Borucki, W. J., et al. 2000, PASP, in preparation

Brown, T. M., \& Kolinski, D. 1999, http://www.hao.ucar.edu/public/research/stare/stare.html

Buffington, A., Booth, C.H., \& Hudson, H.S. 1991 PASP, 103, 685

Butler, R. P., \& Marcy, G.W., Vogt, S.S., \& Apps, K. 1998, PASP, 110, 1389

Charbonneau, D., Brown, T. M., Latham, D. W., \& Mayor, M. 1999, ApJ, 529, L45

Gilliland, R. L. 1992, AJ, 101 No. 2, 541

Guillot, T., Burrows, A., Hubbard, W. B., Lunine, J. I., \& Saumon, D. 1996, ApJ, 459, L35

Gonzalez, G., Wallerstein, G., Saar, S. 1999, ApJ, 511, L111-L114

Henden, A. A., Kaitchuk, R. H., 1990, Astronomical Photometry, (Virginia: WilmannBell)

Henry, G. W., Marcy, G. W., Butler, R. P., \& Vogt, S. S. 1999, ApJ, 529, L41

Janes, K. 1996, JGR, 101 NO. E6, 14,853

Marcy, G. W., Cochran, W. D., \& Mayor, M. 2000, in Protostars and Planets IV, ed. V. Mannings, A. P. Boss, \& S. S. Russell, (Tucson: University of Arizona Press), in press

Mazch, T. ct al. 2000, ApJ, in preparation 
Stone, R. P. S. 1992, University of California Lick Observatory Technical Report No. 61, Nickel Telscope User's Manual

Young, A. T. 1974, Methods of Experimental Physics, Vol. 12A - Astrophysics, Optical and Infrared, (New York: Academic Press) 
Fig. 1.- Data from the night of September 5, 1999 (U. T.) with the residual linear trend removed. The standard deviation of the night's data set was 0.0023 magnitudes, somewliat larger than the formal errors shown as error bars for each data point.Superimposed are simulated idealized transits. Solid line - the central transit of a Jupiter radius planet, long dashed line - central transit of an inflated Jupiter, short dashed line - the grazing transit of Jupiter radius planet, dot-dash line - grazing transit of an inflated Jupiter. All cases except a Jupiter radius grazing transit would have been detected at the $>3$ sigma level per data point. The uncertainties in the ephemeris amount to \pm 2 ticks on the time axis.

Fig. 2.- Data from the night of September 8, 1999 (U. T.) with the residual linear trend removed. The standard deviation of the night's data set was 0.0015 magnitudes, somewhat larger than the formal errors shown as error bars for each data point. Superimposed are simulated idealized transits. Solid line - the central transit of a Jupiter radius planet, long dashed line - central transit of an inflated Jupiter, short dashed line - the grazing transit of Jupiter radius planet, dot-dash line - grazing transit of an inflated Jupiter. All cases except a Jupiter radius grazing transit would have been detected at the $>3$ sigma level per data point. The uncertainties in the ephemeris amount to \pm 2 ticks on the time axis. 
Table 1. Journal of Observations

\begin{tabular}{cccccc}
\hline \hline $\begin{array}{c}\text { Date } \\
\text { (U. T.) }\end{array}$ & $\begin{array}{c}\text { Begin } \\
\text { (U. T.) }\end{array}$ & $\begin{array}{c}\text { End } \\
\text { (U. T. })\end{array}$ & $\begin{array}{c}\text { Exposure } \\
(\mathrm{s})\end{array}$ & $\begin{array}{c}\sigma^{\mathrm{a}} \\
\text { (mag.) }\end{array}$ & $\begin{array}{c}\sigma^{\mathrm{b}} \\
\text { (mag.) }\end{array}$ \\
\hline & & & & & \\
September 5, 1999 & $4: 15$ & $7: 45$ & 250 & 0.0022 & 0.0015 \\
September 8, 1999 & $4: 30$ & $8: 30$ & 250 & 0.0030 & 0.0023 \\
\hline
\end{tabular}

${ }^{\mathrm{a}}$ The measured photometric precision $\sigma$ before the linear trend was removed.

b The measured photometric precision $\sigma$ after the linear trend was removed. 


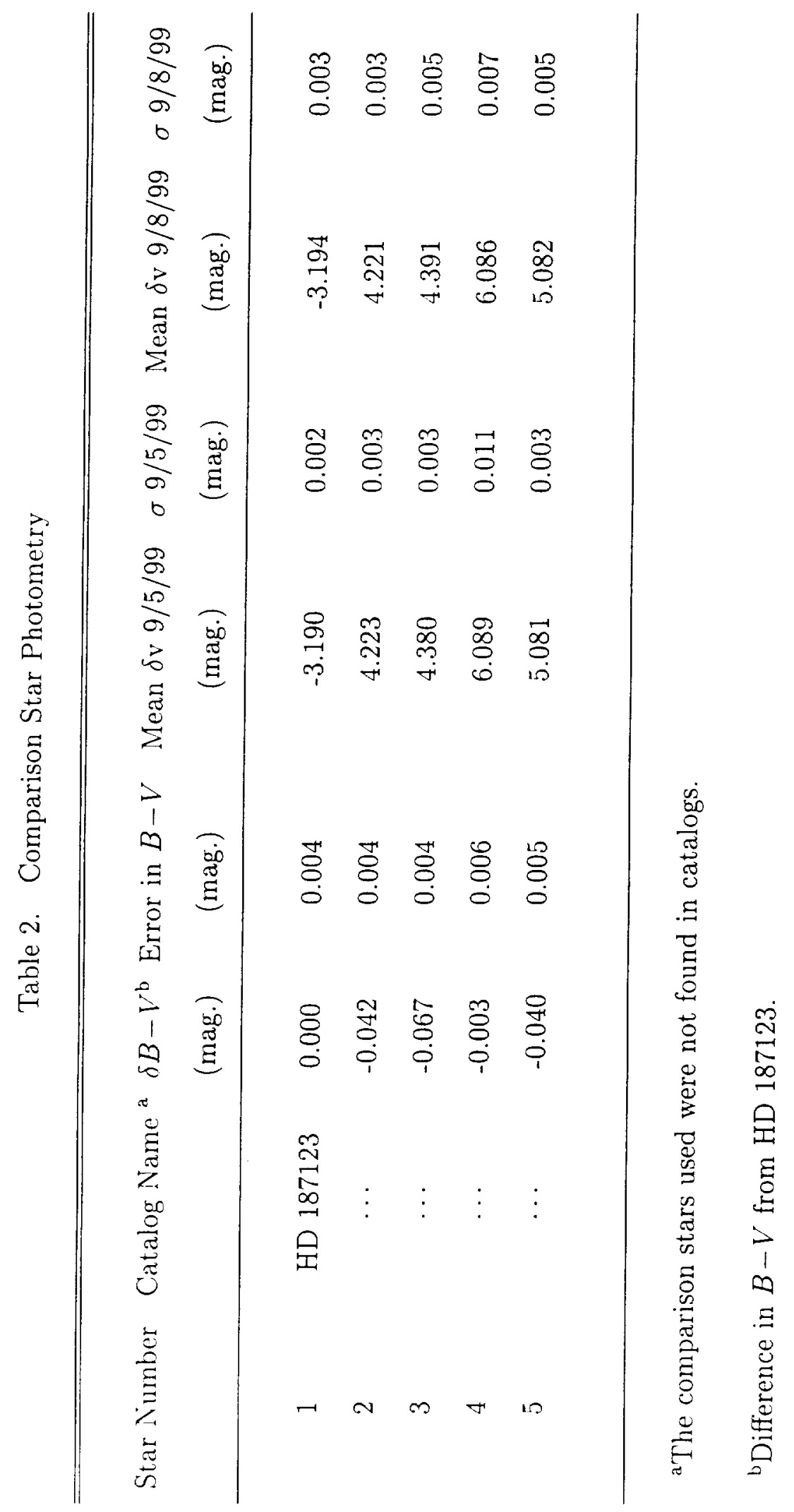


Table 3. HD 187123 Stellar Properties

\begin{tabular}{cr}
\hline \hline & \\
& \\
$M_{\star}\left(M_{\odot}\right)$ & $1.08^{\mathrm{a}}$ \\
$L_{\star}\left(L_{\odot}\right)$ & $1.35^{\mathrm{b}}$ \\
$\mathrm{T}_{\text {eff }}(\mathrm{K})$ & $5830^{\mathrm{a}}$ \\
$R_{\star}\left(R_{\odot}\right)$ & $1.14^{\mathrm{c}}$ \\
$\mathrm{P}_{\text {rot }}($ days $)$ & $30^{\mathrm{b}}$ \\
$v$ sin $i(\mathrm{~km} / \mathrm{s})$ & $<2^{\mathrm{b}}$ \\
\hline & \\
${ }^{\mathrm{a}}$ Gonzalez & et \\
(1996) \\
${ }^{\mathrm{b}}$ Butler et al. $(1998)$ \\
${ }^{\mathrm{c}}$ Stellar adius estimate \\
used to calculate transit \\
depths.
\end{tabular}


Table 4. HD 187123b Properties

\begin{tabular}{cr}
\hline \hline & \\
& \\
$M_{p} \sin i\left(\mathrm{M}_{\mathrm{J}}\right)$ & $0.52^{\mathrm{a}}$ \\
Orbital Semimajor Axis (AU) & $0.0415(0.003)^{\mathrm{a}}$ \\
Period (days) & $3.0097(0.003)^{\mathrm{a}}$ \\
Eccentricity & $0.03(0.03)^{\mathrm{a}}$ \\
Maximum inclination (degrees) & $82.7^{\mathrm{b}}$ \\
\hline
\end{tabular}

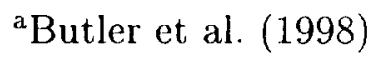

${ }^{b}$ Assuming a planet radius of $1.27 R_{J}$ similar to HD 209458b. 


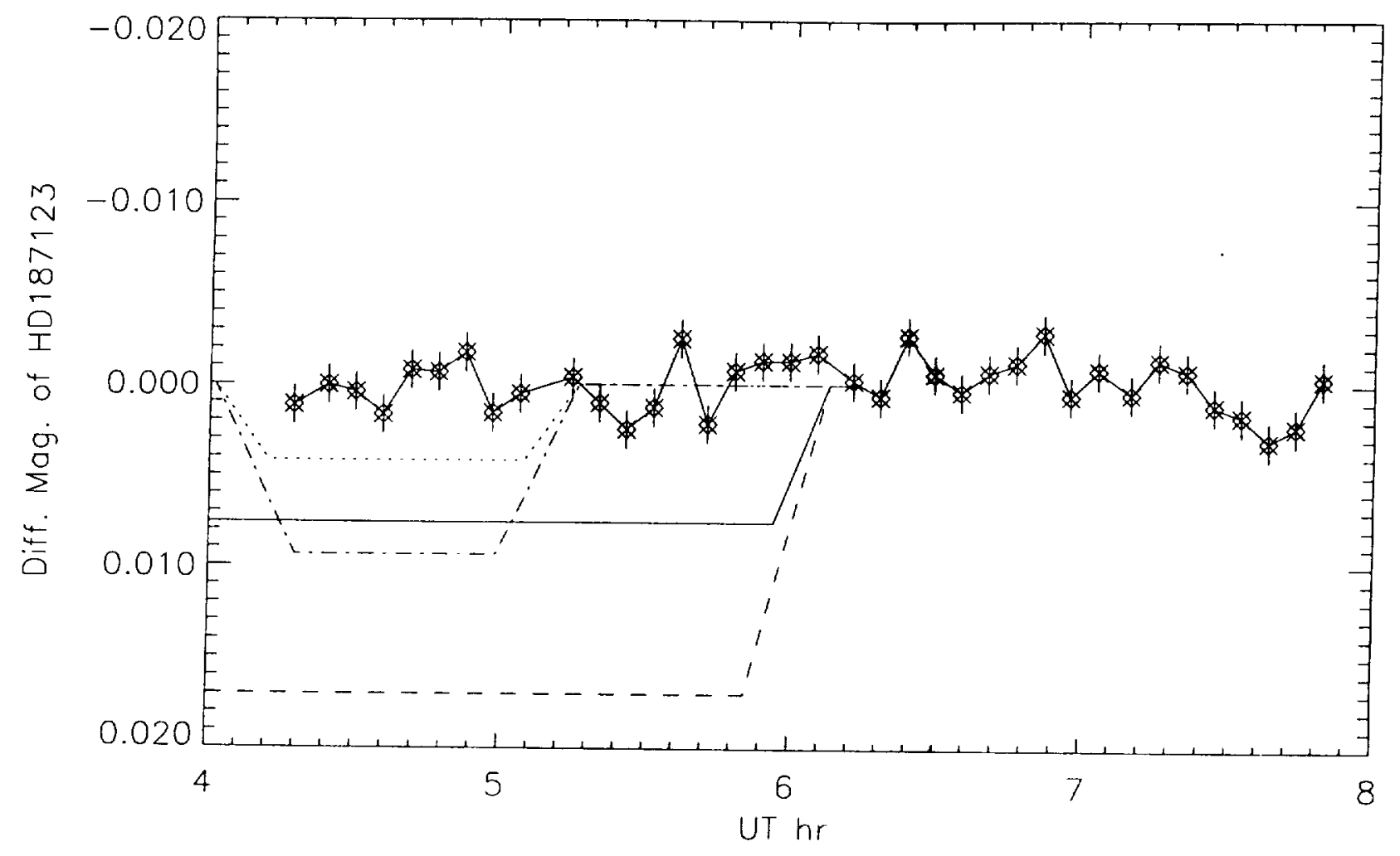




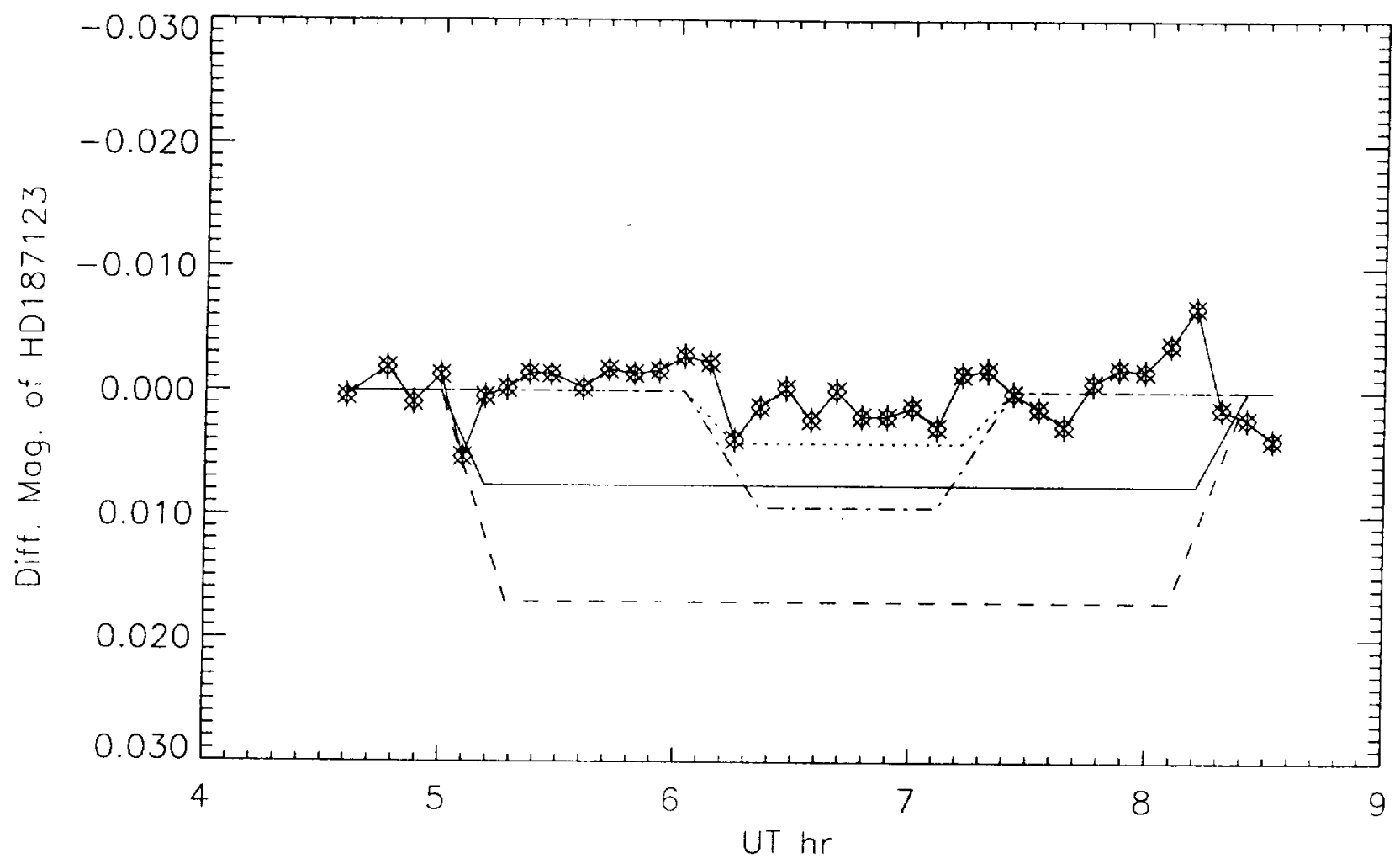

